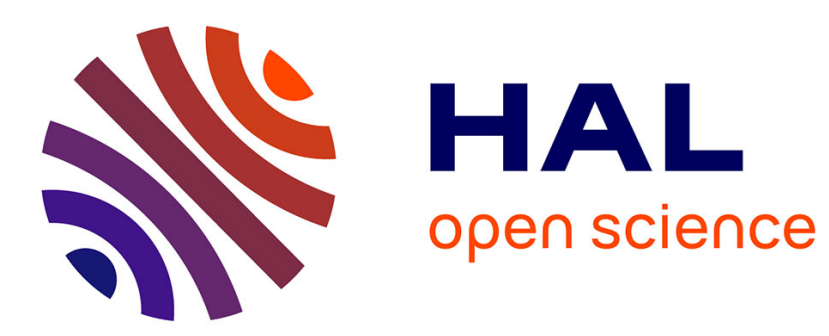

\title{
CCD Positions of Saturn and its Major Satellites from 2002-2006
}

\author{
Qing Yu Peng, Alain Vienne, X. P. Wu, L. L. Gan, Josselin Desmars
}

\section{To cite this version:}

Qing Yu Peng, Alain Vienne, X. P. Wu, L. L. Gan, Josselin Desmars. CCD Positions of Saturn and its Major Satellites from 2002-2006. The Astronomical Journal, 2008, 136 (5), pp.2214-2221. 10.1088/0004-6256/136/5/2214 . hal-00807389

\section{HAL Id: hal-00807389 \\ https://hal.sorbonne-universite.fr/hal-00807389}

Submitted on 3 Apr 2013

HAL is a multi-disciplinary open access archive for the deposit and dissemination of scientific research documents, whether they are published or not. The documents may come from teaching and research institutions in France or abroad, or from public or private research centers.
L'archive ouverte pluridisciplinaire HAL, est destinée au dépôt et à la diffusion de documents scientifiques de niveau recherche, publiés ou non, émanant des établissements d'enseignement et de recherche français ou étrangers, des laboratoires publics ou privés. 


\title{
CCD POSITIONS OF SATURN AND ITS MAJOR SATELLITES FROM 2002-2006
}

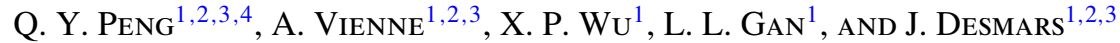 \\ ${ }^{1}$ Department of Computer Science, Jinan University, Guangzhou 510632, China; pengqy@pub.guangzhou.gd.cn \\ ${ }^{2}$ University of Lille, LAL-IMCCE 59000 Lille, France \\ ${ }^{3}$ Institut de Mécanique Céleste et de Calcul des Ephémerides, UMR8028, CNRS, Paris, France \\ ${ }^{4}$ Joint Laboratory for Optical Astronomy, Chinese Academy of Sciences, Kunming 650011, China \\ Received 2008 May 4; accepted 2008 September 10; published 2008 October 15
}

\begin{abstract}
This paper presents 2154 precise positions of Saturn and its major satellites from 359 CCD exposures taken with the $1 \mathrm{~m}$ telescope at the Yunnan Observatory during the years 2002-2006. It also describes the improved image-processing techniques for the pixel positional measurement of Saturn's rings and its major satellites, especially for Mimas and Enceladus. The four bright satellites S3-S6 (i.e., Tethys, Dione, Rhea, and Titan) of Saturn are used to calibrate the CCD field of view by comparing their pixel positions with their theoretical ones from the theory TASS1.7. The observational positions of these major satellites, when measured with respect to Rhea, usually have a good agreement with their theoretical ones except for Mimas, which has the biggest systematic difference of about -0.3 arcsec in R.A. in its 2002 observational data sets. However, these differences of Mimas become much smaller when the recent Jet Propulsion Laboratory ephemeris is replaced. The rms errors in each coordinate are about 40 mas for Saturn and its bright satellites S2-S6, and 90 mas for Mimas. These positional observations are comparable to the best ground-based CCD observations.
\end{abstract}

Key words: astrometry - planets and satellites: general - techniques: image processing

Online-only material: machine readable and VO tables

\section{INTRODUCTION}

Mimas and Enceladus are the two important satellites used to quantify the Saturnian tidal dissipation from its influence on the satellite dynamics. However, observations of these two satellites based on a ground-based telescope are usually much less numerous and less precise than the other major satellites of Saturn. The main causes are their small separations and great brightness differences from their primary planet and rings. In past years, some valuable and quite numerous positions for Enceladus and Mimas with rms errors of 70-90 mas in each coordinate were obtained during the opposition of Saturn in 1995 (Vienne et al. 2001a) beside the occurrence of mutual phenomena of the major satellites of Saturn (Arlot \& Thuillot 1993). In order to get the pixel positions of the observed satellites, Vienne et al. used the software ARSTROL (Colas 1996) based on the adjustment of a point-spread function (PSF). However, the positional precision for Mimas and Enceladus is usually a little worse during the period of the nonmutual phenomena. For example, Mimas had a precision of about 120 mas by Veiga et al. (2003) when the same telescope and procedure were used. Probably, the mutual phenomena of the observed satellites may provide us with better opportunities to obtain precise positions of Mimas and Enceladus. Another example is the observations by Peng et al. (2002); a precision of about 60 mas was derived for Mimas observed in 1996. In order to develop and test the image-processing techniques of Saturn and its satellites, we had used 495 CCD exposures (Peng 2005 , hereafter P1) taken with the $1 \mathrm{~m}$ telescope at the Yunnan Observatory during the years 2002-2004. In detail, we adopted two techniques-edge detection and halo removal- to derive the pixel center of Saturnian rings and quite a clear background for the measured satellites (see Figure 1 in P1). According to Pascu et al. (1987), the background here includes skylight, instrumentally scattered light, electronically generated energy, and, most important to this application, a large contribution from the halo of Saturn and its rings. Our analysis of the intersatellite positions among four bright satellites of Saturn (S3-S6) and of the Saturn-Titan positions showed that the measured positions had almost the same dispersions, that is, 50 and 60 mas in right ascension and declination, respectively. Unfortunately, we also found that for the fainter satellites, Enceladus and Mimas, poor residuals can reach up to 0.1 and $0.2 \mathrm{arcsec}$, respectively, in both directions. To further refine the precision of positional measurement of Saturn and its faint satellites, especially for Mimas, we try to adopt a Laplacian of Gaussian (LoG) algorithm in the present work to detect more accurately the edge of Saturnian rings and a Gaussian fit to the image of a satellite after our former halo-removal processing. Therefore, more precise positions of Saturn and its satellites, especially for Mimas and Enceladus, are expected. This paper is organized as follows. In Section 2, we describe the observations. In Section 3, the improved image-processing techniques are explained for Saturnian rings and faint satellites. Section 4 gives all the results of the calibration parameters. Our observations for the positions of Saturn and its major satellites are described in detail in Section 5, and Section 6 presents the conclusions.

\section{OBSERVATIONS}

During the period of 2002-2006, more than 1000 CCD exposures for Saturn and its major satellites were made with the $1 \mathrm{~m}$ telescope at the Yunnan Observatory (geographical longitude: $\mathrm{E} 102^{\circ} 47^{\prime} .3$, latitude: $\mathrm{N} 25^{\circ} 1{ }^{\prime} .5$, and altitude: $2000 \mathrm{~m})$. The Johnson I-type filter was used while observing. The specifications of the telescope and the CCD receiver that were used can be seen in Table 1. To refine the positional measurement of Mimas and other satellites, we have selected about 400 CCD exposures on which Mimas and Enceladus (together with Saturn and all the other four major satellites) appeared. 
Table 1

Specifications of the $1 \mathrm{~m}$ Telescope and its Attached CCD Chip at the Yunnan Observatory

\begin{tabular}{lc}
\hline \hline Focal length & $1300 \mathrm{~cm}$ \\
$F$-ratio & 13 \\
Diameter of primary mirror & $100 \mathrm{~cm}$ \\
CCD field of view & $6.4 \times 6.4$ \\
Size of pixel & $24 \mu \times 24 \mu$ \\
Size of CCD array & $1024 \times 1024$ \\
Angular extent per pixel & $0^{\prime \prime} .37 /$ pixel \\
\hline
\end{tabular}

For the observations in 2002-2004, the halo distributions of Saturn and its rings usually have good symmetry (see Figure 1 in P1). However, in our latter CCD exposures after 2005 December 1, an annulus "ghost image" appears possibly due to some incorrect positioning of the filter that was being used; see Figure 1 for more details. Obviously, Saturn and its rings are located on this additional and asymmetric light distribution. Therefore, our former halo-removal technique (P1) cannot entirely deal with this situation due to the loss of our reference criterion-symmetric halo distribution about the center of Saturn and its rings. We have to improve this technique to measure the positions of all the major satellites of Saturn. More detailed descriptions will be shown in Section 3. Besides, in order to have almost a uniform precision of calibration parameters, we have selected those CCD exposures on which the four bright satellites S3-S6 (Tethys, Dione, Rhea, and Titan) appear together with Mimas and Enceladus. As a result, we have found 359 exposures in 13 nights. The detailed distributions of CCD exposures observed on different dates can be seen in Table 2.

\section{POSITIONAL MEASURING METHODS}

\subsection{Edge Detection of Saturn's Rings}

In our earlier version of image-processing software, a fourstep procedure is carried out to detect the edge of the Saturnian rings and then a halo-removal algorithm is performed (P1). Now we present some descriptions and a demonstration of this software (see Figures 2(a)-(d)).

Distributions and Solved Parameters of Our Observations

\begin{tabular}{lcccrccc}
\hline \hline Data set & Year & Month & Day & \multicolumn{1}{c}{$N$} & \multicolumn{1}{c}{$P$} & Scale & Orientation \\
\hline 020101 & 2002 & 01 & 01 & 37 & 84 & $0.37385(0.37380)$ & $170.915(170.907)$ \\
$020103 \mathrm{a}$ & 2002 & 01 & 03 & 19 & 62 & $0.37385(0.37381)$ & $173.486(173.479)$ \\
$020103 \mathrm{~b}$ & 2002 & 01 & 03 & 8 & 31 & $0.37389(0.37384)$ & $176.354(176.347)$ \\
020104 & 2002 & 01 & 04 & 72 & 135 & $0.37384(0.37382)$ & $179.510(179.505)$ \\
030108 & 2003 & 01 & 08 & 20 & 106 & $0.37390(0.37389)$ & $179.152(179.152)$ \\
030110 & 2003 & 01 & 10 & 49 & 78 & $0.37383(0.37383)$ & $179.144(179.140)$ \\
040101 & 2004 & 01 & 01 & 21 & 38 & $0.37392(0.37396)$ & $-179.492(-179.500)$ \\
040105 & 2004 & 01 & 05 & 11 & 39 & $0.37386(0.37386)$ & $-179.539(-179.566)$ \\
041214 & 2004 & 12 & 14 & 17 & 60 & $0.37386(0.37379)$ & $178.806(178.798)$ \\
050105 & 2005 & 01 & 05 & 7 & 20 & $0.37380(0.37391)$ & $179.002(178.983)$ \\
051201 & 2005 & 12 & 01 & 6 & 6 & $0.37342(0.37346)$ & $174.888(174.880)$ \\
060112 & 2006 & 01 & 12 & 8 & 78 & $0.37351(0.37350)$ & $178.872(178.872)$ \\
060206 & 2006 & 02 & 06 & 43 & 86 & $0.37361(0.37364)$ & $179.972(179.972)$ \\
060207 & 2006 & 02 & 07 & 41 & 85 & $0.37358(0.37364)$ & $179.988(179.980)$ \\
All & & & & 359 & & & \\
\hline
\end{tabular}

Notes. Column 5 denotes the number of exposures corresponding to the data set of Column 1. Column 6 shows the period of time (unit: min) for each data set during observing. The solved parameters, scale factor (unit: arcsec/pixel), and orientation (unit: degree), are listed in the last two columns (details can be seen in Section 4). 

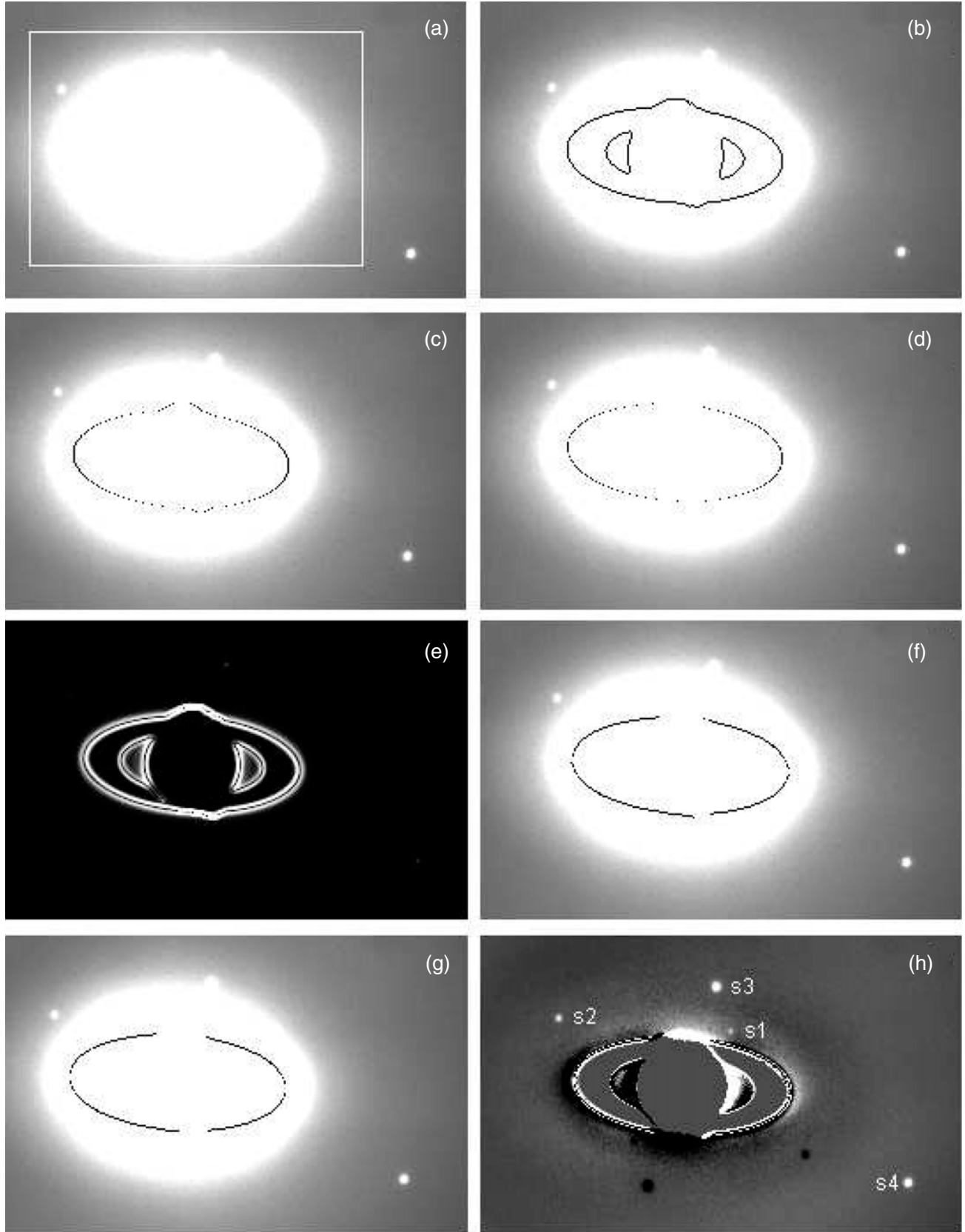

Figure 2. Image-processing procedures for Saturn and its satellites. This illustrative image was taken on 2002 January 4 at the Yunnan Observatory.

obtained. In the process of fitting, some wild points were further rejected. Figure 2(d) shows the residual points on the fitted ellipse. In our former version of the software, halo removal was further carried out with an assumption that halos are distributed symmetrically around the fitted geometric center.

However, the asymmetrical halo in Figure 1 dissatisfies the above assumption. We have to search some new techniques. Among the classical edge-detection algorithms, the edges in the zero-crossing image from LoG are thinner than the gradient edges (Gonzalez \& Woods 1992; Figure 2(e): an image after LoG processing for the original image) and can remove the smooth structure in the image. Thus, LoG can be suitable not only to avoid mostly the asymmetric halo (up to a polynomial of degree 2), but also to detect a sharper and finer edge of Saturn's rings. In practice, we simply adjust the abovementioned coefficient $k$ in Equation (1) and let the fitted ellipse edge obtained in Step 4 act as a reference for further searching the LoG edge point of the outer rings of Saturn. During the search, some saturated pixel points were also rejected. Figure 2(f) shows the derived LoG edge points. Similar to Step 4, we fit these LoG edge points iteratively to an ellipse by least squares and reject some large residual points (Figure $2(\mathrm{~g})$ ). Finally, we find that even slightly-blooming images are qualified to detect the pixel center of Saturn. After a halo removal is carried out, more opportunity for faint satellites, such as Mimas and Enceladus, can be obtained (Figures 2(h) and 3). It should be noted that for the observational sets in 2005 and 2006, since some asymmetry appears from the "ghost image" as mentioned in Section 2, the residual halo still exists after our halo-removal processing (comparing Figure 2(h) with Figure 3 ) although the level of the residual halo becomes much lower. Figure 4 quantifies the gray-level distributions for Mimas (S1) in Figure 3, 


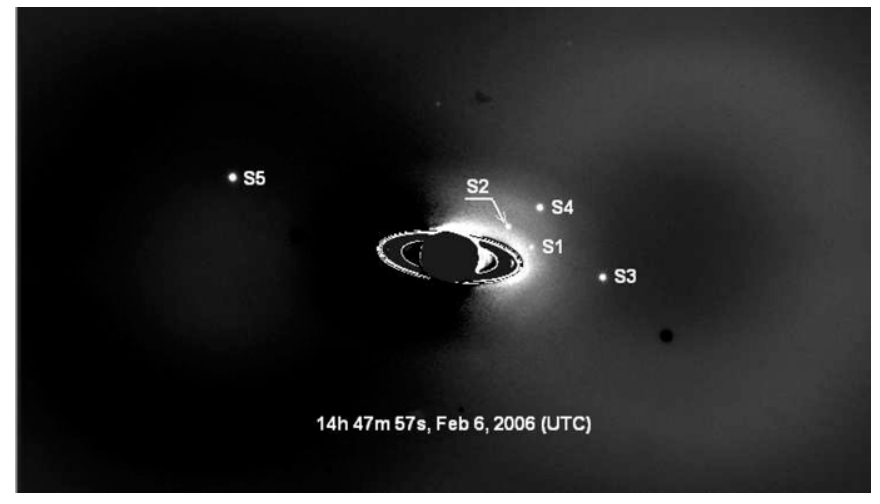

Figure 3. An asymmetric halo resides after the LoG edge detection and halo removal for the image in Figure 1.

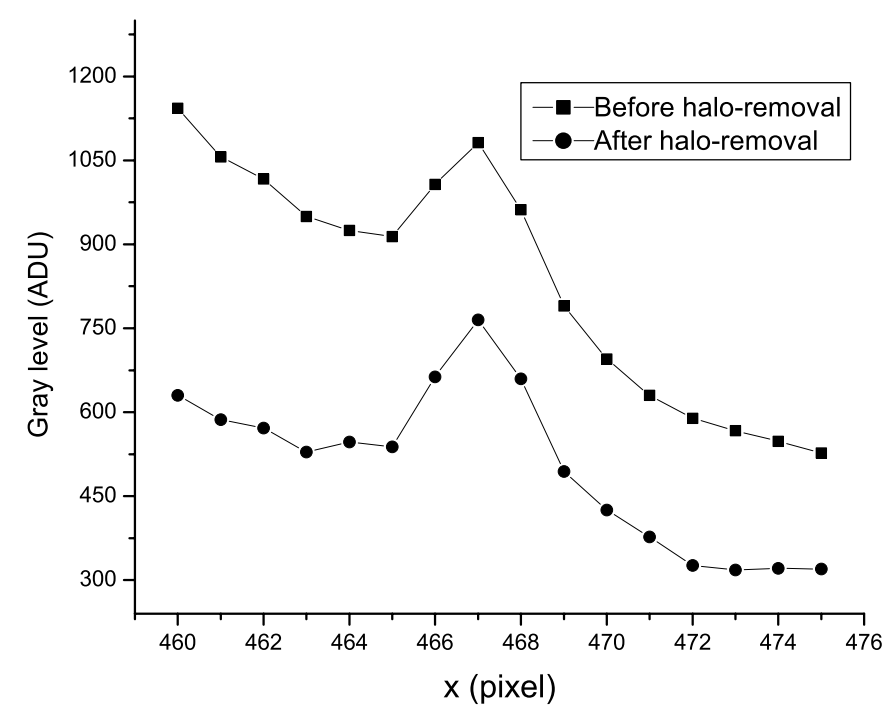

Figure 4. Gray-level distribution along the horizontal line through Mimas (S1) in Figure 3, before and after its halo removal.

along the horizontal line through its measured pixel center before and after the halo removal. Obviously, a Gaussian with a first-order polynomial background will be more appropriate to fit in order to derive an accurate position of the faint satellite Mimas (see the next subsection for more details).

\subsection{Pixel Position of Satellites}

After the halo removal, we adopted a two-dimensionmodified moment algorithm (Peng et al. 2002; P1) for the pixel positional measurement of Saturnian major satellites. This algorithm usually has good precision for a bright satellite. However, the new characteristic appearing in 2005 and 2006's observational images forces us to find another method to measure the centroids of Mimas and Enceladus. Pascu et al. (1987) represented their Uranian image as a two-dimensional symmetric Gaussian sitting on a sloping background surface, which was quadratic in $x$ and $y$. A similar practice is carried out for our present CCD images. In detail, we use the following formula to model the gray-level distribution including a satellite:

$$
\begin{gathered}
E(x, y)=a+b x+c y+d x^{2}+e x y+f y^{2} \\
+H e^{-\left[\left(x-x_{0}\right)^{2}+\left(y-y_{0}\right)^{2}\right] / 2 \sigma^{2}}
\end{gathered}
$$

where $\sigma$ is the Gaussian radius parameter and $\left(x_{0}, y_{0}\right)$ are the coordinates of the center of the Gaussian function. For

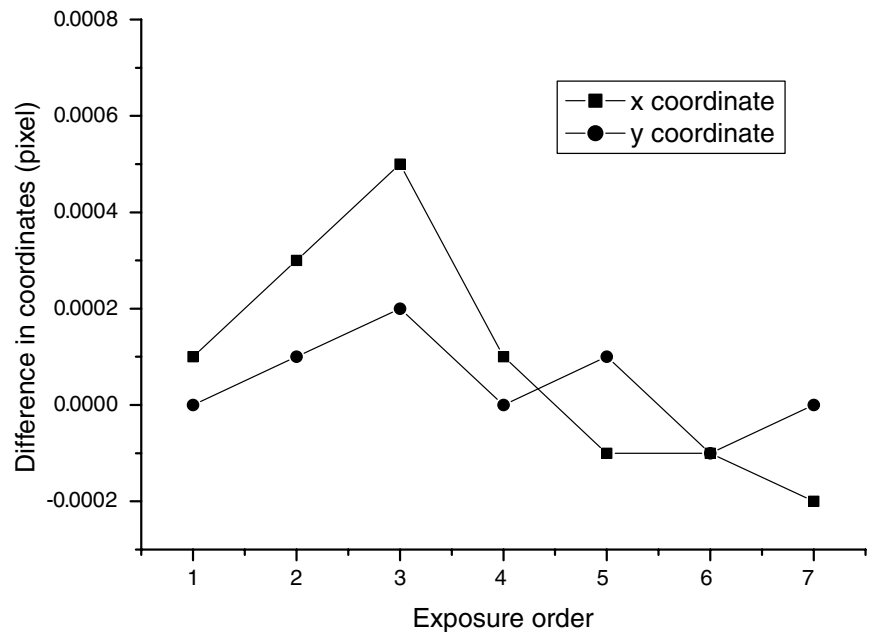

Figure 5. Pixel-coordinate differences in the sense of second-order minus firstorder background Gaussian fit for seven continuous exposures.

comparison, we also adopt a sloping background surface that is linear in $x$ and $y$,

$$
E(x, y)=a+b x+c y+H e^{-\left[\left(x-x_{0}\right)^{2}+\left(y-y_{0}\right)^{2}\right] / 2 \sigma^{2}} .
$$

In our measurement, we find that only a very small difference (usually less than 0.01 pixel) exists when the above two models are tested. For example, Figure 5 shows the difference between measured pixel coordinates using the two models for the first seven continuous exposures from the data set 060206. For simplicity, a linear sloping background surface was adopted in our final measurement for the observations after (and including) 2005 December 1. But only a two-dimensional symmetric Gaussian sitting on a flat background surface is used for the other satellite images before this date, simply because on the whole, good symmetry appears on their background distributions after our halo removal.

\section{CALIBRATIONS}

At present, two well-known ephemerides, TASS1.7 (Duriez \& Vienne 1997; Vienne \& Duriez 1995) and JPL ephemeris, ${ }^{5}$ are available for comparison with our CCD observations. Note that, TASS1.7, unlike JPL ephemeris, is not only an ephemeris as its analytical formulae show, but also allows theoretical studies on the dynamics of this system, such as tidal effects and secondary resonances. However, JPL ephemeris has been increasingly improved by using the newest observations from instruments based in space (such as the Hubble Space Telescope (HST) and Cassini spacecraft) and on the ground. Therefore, it will be interesting to compare the two theories, especially through observations. To compare the pixel positions of all measured objects (Saturn and its satellites) with their theoretical positions, the calibration of CCD fields of view (FOVs) is essential. Usually, there are rare stars or no stars appearing in our CCD FOVs because of their small size (about 400 arcsec on each side) and short exposure time (1-3 s) for each CCD exposure. The four major satellites-Tethys, Dione, Rhea, and Titan-have been used to calibrate by several authors (e.g., Harper et al. 1997; Qiao et al. 1999; Shen et al. 2001; Vienne et al. 2001a, 2001b; Peng et al. 2002; P1) because these satellites

\footnotetext{
5 http://ssd.jpl.nasa.gov/
} 

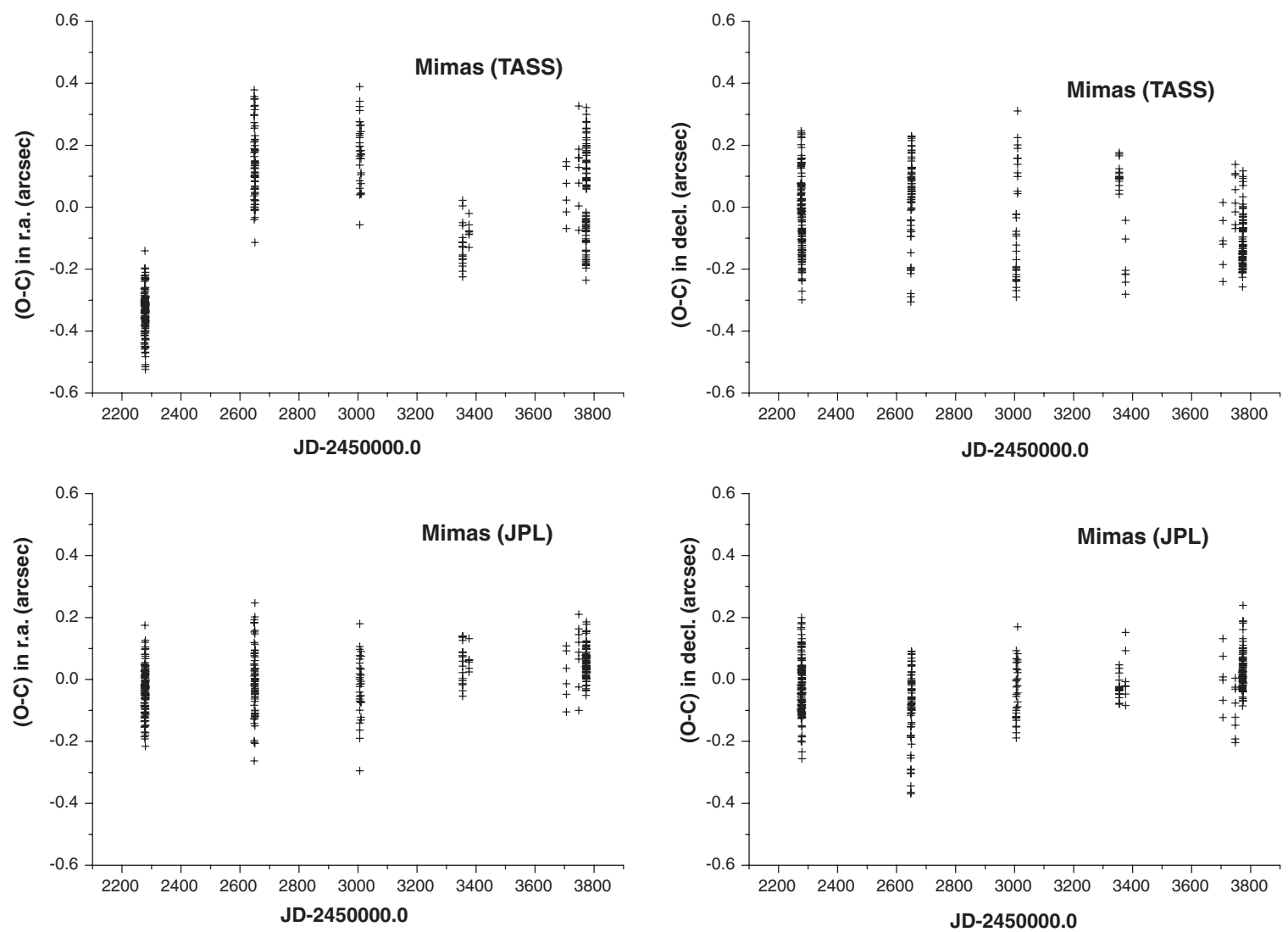

Figure 6. $(\mathrm{O}-\mathrm{C})$ residuals of Mimas when TASS1.7 and JPL ephemeris are adopted.

have the best ephemerides and are thus probably affected by the smallest systematic effects. In detail, for a series of CCD exposures, we suppose that each FOV has two parameters, a scale factor and an orientation angle (the angle between the $y$-axis of the CCD camera and the true longitude). Theoretically, the position of the tangential point of each CCD plane on the celestial sphere needs to be precisely known to compute absolute and precise positions of measured objects. But we do not know where the tangential point is in our CCD FOV. Besides, relative positions of intersatellites (or satellite-Saturn) can also be used to improve the ephemerides of satellites. Therefore, the position of the tangential point on the celestial sphere does not need to be known very precisely and does not need to be solved for as an independent adjustment parameter. Detailed explanations can be seen in the literature by Vienne et al. (2001a, 2001b). Our calibration of the used CCD FOV is done in the same way as was done for the former one in P1. Specifically, we obtained the theoretical (geocentric-apparent) positions of all our six satellites from TASS1.7 and of Saturn from the JPL ephemeris DE405 (Standish 1998), and then transformed them into positions that are comparable to pixel positions of these satellites and of Saturn by taking all the astrometric effects (such as atmospheric refraction, diurnal parallax, diurnal aberration, and central projection) into account. For computing refraction correction, only normal condition formulae are required. For the other astrometric effects, one can see Vienne et al. (2001a, 2001b) for more details. Besides, the difference in the scale factor between the two coordinate directions of the CCD being used has been tested (Vienne et al. 2001b). We found that this difference was within the probable error. Theoretically, the best way to test is by imaging a star cluster using varying orientation angles. For our present observations, only a simple model with two parameters (scale factor and orientation) is adopted. Table 2 lists the scale and orientation solved for each data set. As a comparison, the JPL ephemeris is also used to calibrate the above-mentioned four satellites. In Table 2, the numbers in the parentheses correspond to the calculations using the JPL ephemeris. One can see only a very small difference when the two ephemerides are adopted. We estimate that the positional errors in our observations from the two sets of calibration parameters are usually less than 0.02 arcsec in each coordinate. Note that two suits of parameters on 2002 January 3 resulted from a mechanical problem while observing.

\section{POSITIONS OF SATURN AND ITS SATELLITES}

Rhea or Titan is usually adopted as a reference object to measure the relative position of the other major satellites. For example, Vienne et al. (2001a) and Veiga et al. (2003) usually adopted Rhea, but Peng et al. (2002) and Qiao et al. (1999) mainly adopted Titan as their reference object. Theoretically, if the measured satellite (such as Mimas) has greater separation from the referenced object, its positional precision will be slightly worse due to the atmospheric effects (Lindegren 1980). Besides, a greater separation will encounter more systematic error from the calibration parameters that are determined. Therefore, in this paper, we prefer to choose Rhea as the reference object for the data analysis of Saturn and the other satellites. Table 3 lists the statistics of all our observations of Saturn and its major satellites in 2002-2006 for each observational data set or the whole data. Here, the theory of TASS1.7 is once more referred to for computing the theoretical positions 

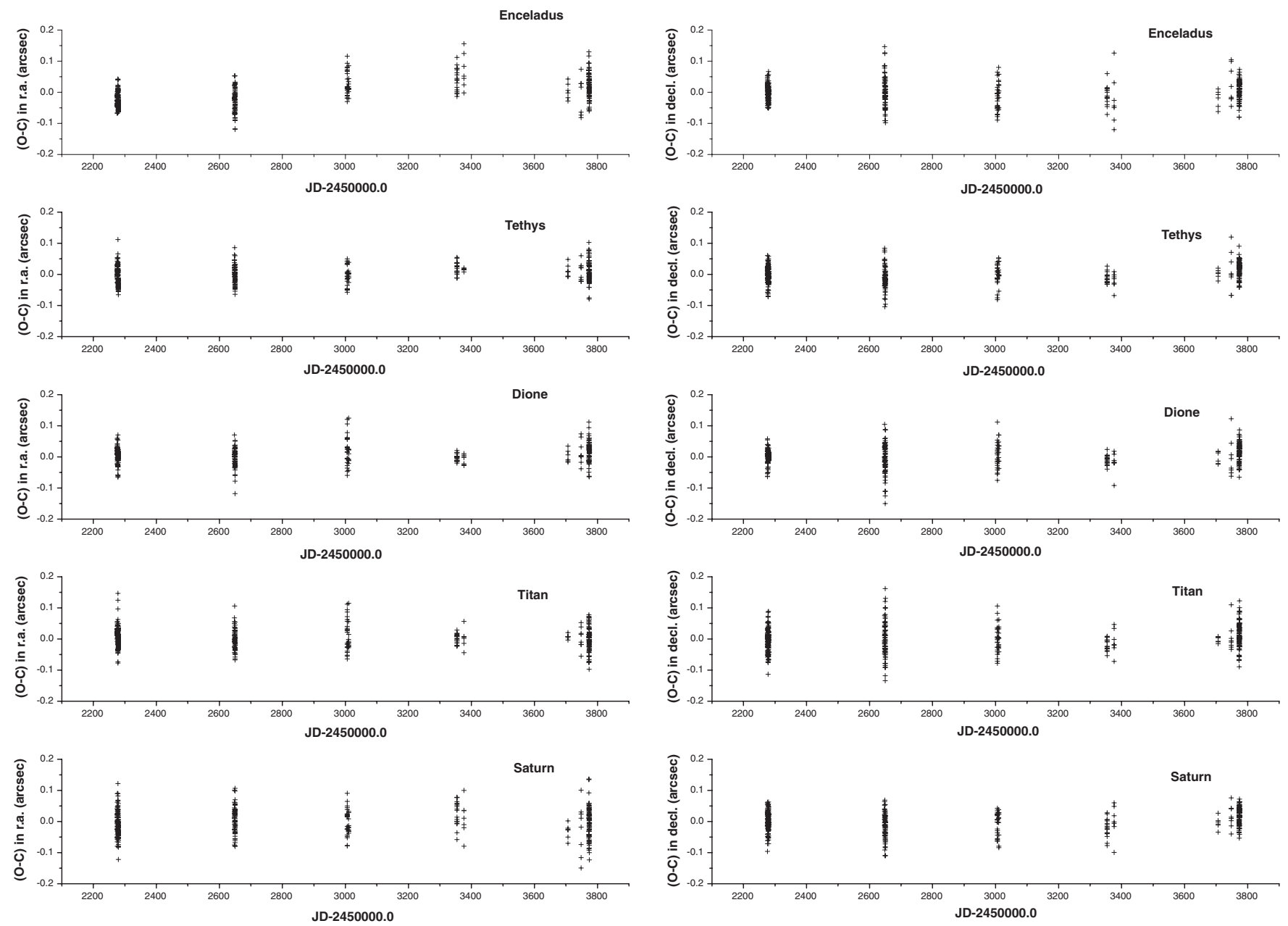

Figure 7. (O-C) residuals of Enceladus, Tethys, Dione, Titan, and Saturn when JPL ephemerides are referred.

of all satellites. As a comparison, the JPL ephemeris is also used to do the same computations. We can see that a small residual (30-40 mas in each coordinate) is usually observed for the bright satellites-Tethys, Dione, and Titan. Saturn is once again confirmed to have a good precision comparable to these bright satellites and has no systematic offset in the statistical sense from its theory. Of course, the observed minus computed $(\mathrm{O}-\mathrm{C})$ for Saturn is only the reflection of its center positions when measured with respect to Rhea, and is obviously internal. Furthermore, the measured precision for Enceladus is also significantly improved by a factor of 2 when compared with our former measurements (P1) and especially when JPL ephemeris is referred. However, there are obvious systematic differences for Mimas, especially in its 2002 observations when TASS 1.7 was adopted for its theoretical positions in right ascension. If the recent JPL ephemeris of Mimas (SAT286 $\times 1$ ) is replaced, the residual improvement is satisfactory. Thus, these observations reveal that there is probably a problem with the theory of Mimas for TASS1.7. Figure 6 shows the distributions of all $(\mathrm{O}-\mathrm{C})$ residuals of Mimas when TASS1.7 and JPL ephemeris are adopted. Figure 7 shows the $(\mathrm{O}-\mathrm{C})$ residual distributions of other major satellites (Tethys, Dione, and Titan) and Saturn when measured with respect to Rhea. Here, only JPL ephemeris is adopted for the theoretical positions of these major satellites in Figure 7. Our observations are the geocentric-apparent coordinates. Table 4 lists an extract of these observations, which are satellite-Saturn positions expressed in arcsec with the corresponding scale and orientation (from JPL ephemeris) in Table 2. The dates correspond to the midtime of the exposure and are not light-time corrected. Specifically, our observations are similar to those in Vienne et al. (2001a) or Peng et al. (2002). Here is a brief description of the content of each record:

1. ID1: the data set identification designed in Table 2 .

2. ID2: the exposure identification from 1 to 359 .

3. year, m, day: year, month and utc date of the observation (NOT light-time corrected).

4. obs: IAU observatory code from the Minor Planet Center.

5. t: observation type. Here $0=\alpha, \delta$, and $1=\Delta \alpha \cos \delta, \Delta \delta$.

6. obj: subject satellite and reference satellite. 0 for Saturn, 1 to 6 for S1 to S6, C for the center of the frame that is not a physical object.

7. obs1, obs2: the first and second coordinates with the unit of arcsec.

8. s1, s2: the estimated errors with the unit of arcsec for obs1 and obs2, respectively. Here, errors from both pixel positions and calibration parameters are taken into account. In the lines of $\mathrm{C}^{*}$ and $\mathrm{C} 0, \mathrm{~s} 1$ and $\mathrm{s} 2$ are all designed 0.00 .

9. s: reference system. Here, $1=$ true equator and equinox of the date of observation.

10. f: reference frame. Here, $1=$ Geocentric and $0=$ Topocentric.

11. $\mathrm{O}-\mathrm{C} 1, \mathrm{O}-\mathrm{C} 2$ : residuals of observations in arcsec when JPL ephemeris is referred. 
Table 3

The Mean $(\mathrm{O}-\mathrm{C}) \mathrm{s}$ (Observed Minus Computed) and their Standard Deviations SD (in arcsec) of Saturn and its Satellites

\begin{tabular}{|c|c|c|c|c|c|c|c|c|c|c|}
\hline Object & $N$ & $\langle\triangle \alpha \cos \delta\rangle$ & SD & $\langle\triangle \delta\rangle$ & SD & $N$ & $\langle\Delta \alpha \cos \delta\rangle$ & SD & $\langle\triangle \delta\rangle$ & SD \\
\hline & & & 020101 & & & & & 020103a & & \\
\hline Mimas & & $-0.335(-0.063)$ & $0.062(0.061)$ & $0.057(-0.038)$ & $0.076(0.077)$ & & $-0.301(0.008)$ & $0.077(0.081)$ & $0.128(0.083)$ & $0.070(0.072)$ \\
\hline Enceladus & & $-0.037(-0.029)$ & $0.016(0.016)$ & $-0.003(0.011)$ & $0.026(0.026)$ & & $0.058(0.000)$ & $0.023(0.024)$ & $0.014(0.000)$ & $0.022(0.022)$ \\
\hline Tethys & 37 & $-0.013(0.010)$ & $0.020(0.020)$ & $0.008(0.004)$ & $0.021(0.021)$ & 19 & $-0.008(0.019)$ & $0.032(0.031)$ & $-0.036(-0.016)$ & $0.034(0.034)$ \\
\hline Dione & & $0.005(0.017)$ & $0.020(0.020)$ & $-0.003(0.003)$ & $0.029(0.029)$ & & $0.006(-0.013)$ & $0.038(0.038)$ & $-0.019(-0.003)$ & $0.025(0.025)$ \\
\hline Titan & & $-0.001(0.024)$ & $0.014(0.014)$ & $-0.000(-0.005)$ & $0.026(0.026)$ & & $-0.006(-0.001)$ & $0.061(0.061)$ & $-0.011(-0.011)$ & $0.054(0.054)$ \\
\hline \multirow[t]{2}{*}{ Saturn } & & $-0.004(-0.004)$ & $0.038(0.038)$ & $0.016(0.003)$ & $0.035(0.035)$ & & $0.029(0.034)$ & $0.041(0.042)$ & $-0.015(-0.005)$ & $0.031(0.031)$ \\
\hline & & & 020103b & & & & & 020104 & & \\
\hline Mimas & & $-0.332(0.018)$ & $0.029(0.029)$ & $-0.059(-0.031)$ & $0.082(0.075)$ & & $-0.350(-0.030)$ & $0.067(0.067)$ & $-0.117(-0.042)$ & $0.071(0.076)$ \\
\hline Enceladus & & $0.048(0.011)$ & $0.008(0.007)$ & $0.017(-0.001)$ & $0.019(0.020)$ & & $0.037(-0.038)$ & $0.017(0.016)$ & $-0.035(-0.006)$ & $0.024(0.024)$ \\
\hline Tethys & 8 & $0.004(0.027)$ & $0.010(0.010)$ & $-0.006(0.016)$ & $0.033(0.033)$ & 72 & $0.006(-0.033)$ & $0.013(0.013)$ & $0.003(-0.003)$ & $0.022(0.022)$ \\
\hline Dione & & $0.020(0.000)$ & $0.018(0.018)$ & $-0.014(0.007)$ & $0.014(0.013)$ & & $-0.005(0.003)$ & $0.008(0.008)$ & $-0.015(0.001)$ & $0.010(0.010)$ \\
\hline Titan & & $0.006(0.007)$ & $0.047(0.046)$ & $-0.003(-0.000)$ & $0.045(0.045)$ & & $-0.003(-0.003)$ & $0.021(0.021)$ & $-0.004(-0.005)$ & $0.031(0.031)$ \\
\hline Saturn & & $0.007(0.010)$ & $\begin{array}{c}0.027(0.026) \\
\mathbf{0 3 0 1 0 8}\end{array}$ & $0.012(0.023)$ & $0.021(0.021)$ & & $-0.020(-0.029)$ & $\begin{array}{c}0.027(0.027) \\
\mathbf{0 3 0 1 1 0}\end{array}$ & $-0.007(0.002)$ & $0.027(0.027)$ \\
\hline Mimas & & $0.179(-0.002)$ & $0.135(0.140)$ & $-0.119(-0.206)$ & $0.113(0.107)$ & & $0.106(-0.009)$ & $0.096(0.090)$ & $0.099(-0.034)$ & $0.069(0.068)$ \\
\hline Enceladus & & $-0.005(-0.020)$ & $0.038(0.034)$ & 0.088( 0.049) & $0.049(0.049)$ & & $-0.087(-0.024)$ & $0.042(0.041)$ & $-0.048(-0.017)$ & $0.037(0.037)$ \\
\hline Tethys & 20 & $-0.036(0.021)$ & $0.026(0.026)$ & $0.006(0.008)$ & $0.050(0.050)$ & 49 & $-0.034(-0.011)$ & $0.026(0.026)$ & $-0.016(-0.019)$ & $0.025(0.025)$ \\
\hline Dione & & $-0.005(0.009)$ & $0.022(0.022)$ & $-0.014(0.009)$ & $0.038(0.038)$ & & $0.011(-0.007)$ & $0.033(0.033)$ & $-0.025(-0.012)$ & $0.052(0.052)$ \\
\hline Titan & & $-0.014(0.014)$ & $0.036(0.036)$ & $0.001(0.004)$ & $0.034(0.034)$ & & $-0.023(-0.008)$ & $0.030(0.030)$ & $0.001(-0.002)$ & $0.065(0.065)$ \\
\hline Saturn & & $-0.003(0.016)$ & $\begin{array}{c}0.036(0.036) \\
\mathbf{0 4 0 1 0 1}\end{array}$ & $0.009(0.013)$ & $0.039(0.039)$ & & $0.015(0.004)$ & $\begin{array}{c}0.045(0.044) \\
\mathbf{0 4 0 1 0 5}\end{array}$ & $-0.024(-0.023)$ & $0.037(0.037)$ \\
\hline Mimas & & $0.202(-0.025)$ & $0.108(0.112)$ & $-0.168(-0.070)$ & $0.084(0.083)$ & & $0.143(-0.024)$ & $0.074(0.074)$ & $0.153(0.018)$ & $0.078(0.075)$ \\
\hline Enceladus & & $-0.041(0.032)$ & $0.044(0.043)$ & $-0.020(-0.027)$ & $0.041(0.041)$ & & $-0.005(0.022)$ & $0.027(0.027)$ & & $0.042(0.042)$ \\
\hline Tethys & 21 & $-0.006(-0.002)$ & $0.036(0.036)$ & $-0.025(-0.005)$ & $0.035(0.035)$ & 11 & $-0.002(-0.000)$ & $0.017(0.017)$ & $-0.012(0.013)$ & $0.032(0.032)$ \\
\hline Dione & & 0.014(0.023) & $0.049(0.048)$ & $-0.032(-0.000)$ & $0.043(0.043)$ & & $0.020(0.011)$ & $0.043(0.043)$ & $0.018(0.021)$ & $0.035(0.035)$ \\
\hline Titan & & $0.009(0.016)$ & $0.053(0.053)$ & $-0.031(-0.001)$ & $0.049(0.049)$ & & $0.002(0.005)$ & $0.044(0.044)$ & $-0.009(0.002)$ & $0.033(0.033)$ \\
\hline Saturn & & $-0.010(0.002)$ & $\begin{array}{c}0.046(0.046) \\
\mathbf{0 4 1 2 1 4}\end{array}$ & $-0.014(-0.006)$ & $0.035(0.035)$ & & $-0.013(-0.012)$ & $\begin{array}{c}0.025(0.025) \\
\mathbf{0 5 0 1 0 5}\end{array}$ & $-0.011(-0.004)$ & $0.044(0.044)$ \\
\hline Mimas & & $-0.124(0.051)$ & $0.070(0.064)$ & $0.105(-0.027)$ & $0.037(0.035)$ & & $-0.077(0.062)$ & $0.033(0.034)$ & $-0.186(0.009)$ & $0.083(0.083)$ \\
\hline Enceladus & & $-0.025(0.041)$ & $0.036(0.037)$ & $-0.016(-0.008)$ & $0.030(0.030)$ & & $-0.024(0.069)$ & $0.056(0.056)$ & $-0.034(-0.024)$ & $0.081(0.082)$ \\
\hline Tethys & 17 & $-0.002(0.019)$ & $0.020(0.020)$ & $-0.018(-0.012)$ & $0.017(0.017)$ & 7 & $0.012(0.016)$ & $0.004(0.004)$ & $-0.021(-0.020)$ & $0.025(0.025)$ \\
\hline Dione & & $0.008(-0.002)$ & $0.012(0.012)$ & $-0.005(-0.011)$ & $0.016(0.016)$ & & $-0.007(-0.008)$ & $0.017(0.016)$ & $-0.018(-0.019)$ & $0.035(0.035)$ \\
\hline Titan & & $-0.012(0.002)$ & $0.015(0.015)$ & $-0.009(-0.018)$ & $0.019(0.019)$ & & $0.003(0.003)$ & $0.030(0.030)$ & $-0.009(-0.008)$ & $0.040(0.040)$ \\
\hline \multirow[t]{2}{*}{ Saturn } & & $0.036(0.025)$ & $0.039(0.040)$ & $-0.021(-0.020)$ & $0.030(0.030)$ & & $-0.006(0.010)$ & $0.056(0.056)$ & 0.004( 0.001) & $0.053(0.053)$ \\
\hline & & & 051201 & & & & & & & \\
\hline Mimas & & $0.049(0.011)$ & $0.085(0.083)$ & $-0.113(0.004)$ & $0.092(0.092)$ & & $0.121(0.084)$ & $0.121(0.102)$ & $0.035(-0.099)$ & $0.079(0.079)$ \\
\hline Enceladus & & $-0.038(0.005)$ & $0.026(0.026)$ & $-0.017(-0.020)$ & $0.028(0.028)$ & & $0.066(0.000)$ & $0.063(0.064)$ & $0.028(0.024)$ & $0.059(0.059)$ \\
\hline Tethys & 6 & $0.017(0.014)$ & $0.021(0.021)$ & $0.002(0.002)$ & $0.014(0.014)$ & 8 & $0.047(0.008)$ & $0.030(0.030)$ & $0.037(0.011)$ & $0.065(0.064)$ \\
\hline Dione & & $-0.018(0.003)$ & $0.020(0.020)$ & $-0.024(-0.001)$ & $0.020(0.020)$ & & $0.038(0.015)$ & $0.039(0.039)$ & $0.016(-0.003)$ & $0.062(0.062)$ \\
\hline Titan & & $-0.008(0.010)$ & $0.009(0.009)$ & $-0.022(-0.002)$ & $0.010(0.010)$ & & 0.013( 0.003) & $0.035(0.035)$ & $0.019(0.005)$ & $0.046(0.046)$ \\
\hline \multirow[t]{2}{*}{ Saturn } & & $-0.048(-0.032)$ & $0.025(0.025)$ & $-0.010(-0.004)$ & $0.020(0.020)$ & & $-0.009(-0.024)$ & $0.084(0.084)$ & $0.027(0.017)$ & $0.037(0.036)$ \\
\hline & & & 060206 & & & & & & & \\
\hline Mimas & & $-0.100(0.047)$ & $0.058(0.046)$ & $-0.167(0.008)$ & $0.037(0.038)$ & & $0.161(0.062)$ & $0.073(0.050)$ & $-0.059(0.056)$ & $0.080(0.073)$ \\
\hline Enceladus & & $0.083(0.035)$ & $0.043(0.042)$ & $0.002(-0.004)$ & $0.032(0.032)$ & & $0.012(0.000)$ & $0.026(0.027)$ & 0.008( 0.019) & $0.024(0.024)$ \\
\hline Tethys & 43 & $0.024(0.022)$ & $0.039(0.039)$ & $0.003(0.013)$ & $0.032(0.031)$ & 41 & $-0.011(-0.002)$ & $0.019(0.019)$ & $0.020(0.020)$ & $0.019(0.019)$ \\
\hline Dione & & $0.017(0.015)$ & $0.039(0.039)$ & $0.010(-0.002)$ & $0.029(0.029)$ & & $0.006(0.023)$ & $0.013(0.013)$ & $0.025(0.032)$ & $0.019(0.020)$ \\
\hline Titan & & $0.004(0.002)$ & $0.048(0.048)$ & $0.002(0.001)$ & $0.040(0.040)$ & & $-0.011(-0.002)$ & $0.019(0.019)$ & $0.020(0.026)$ & $0.039(0.039)$ \\
\hline \multirow[t]{2}{*}{ Saturn } & & $-0.002(0.003)$ & $0.054(0.054)$ & $-0.001(0.007)$ & $0.026(0.026)$ & & $-0.039(-0.016)$ & $0.039(0.039)$ & $0.027(0.029)$ & $0.024(0.024)$ \\
\hline & & & & & & & & & & \\
\hline Mimas & & $-0.085(0.003)$ & $0.233(0.086)$ & $-0.037(-0.024)$ & $0.132(0.094)$ & & & & & \\
\hline Enceladus & & $0.003(-0.005)$ & $0.062(0.043)$ & $-0.009(0.000)$ & $0.046(0.037)$ & & & & & \\
\hline Tethys & 359 & $-0.004(0.000)$ & $0.031(0.031)$ & $-0.002(0.000)$ & $0.032(0.031)$ & & & & & \\
\hline Dione & & $0.006(0.007)$ & $0.029(0.029)$ & $-0.007(0.003)$ & $0.036(0.034)$ & & & & & \\
\hline Titan & & $-0.005(0.003)$ & $0.034(0.034)$ & $-0.001(0.000)$ & $0.043(0.042)$ & & & & & \\
\hline Saturn & & $-0.006(-0.004)$ & $0.045(0.044)$ & $-0.002(0.002)$ & $0.035(0.035)$ & & & & & \\
\hline
\end{tabular}

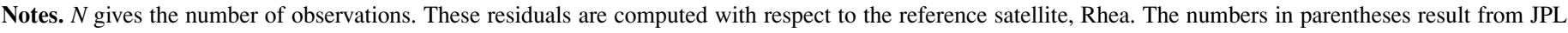
ephemerides $($ SAT2 $86 \times 1)$ for theoretical positions.

12. $r$ : the number of satellites or Saturn used as reference in the computation of $(\mathrm{O}-\mathrm{C})$. Here, $0=$ Saturn.

13. se: the number of series, for the scale factor and orientation corresponding to each series, one can find in Table 2.
14. xpix, ypix: original coordinates in pixels in the sense of $x_{s}-x_{r}$ and $y_{s}-y_{r}$, respectively.

Note that obs 1 and obs 2 for the line including $C^{*}$ refer to the positions (unit: degree) in the celestial coordinate system for the 
Table 4

Extract of the Observations

\begin{tabular}{|c|c|c|c|c|c|c|c|c|c|c|c|c|c|c|c|c|c|c|c|}
\hline ID1 & ID2 & Year & M & Day(utc) & Obs & $\mathrm{t}$ & Obj & Obs1 & Obs2 & s1 & s2 & $\mathrm{s}$ & $\mathrm{f}$ & $\mathrm{O}-\mathrm{C} 1$ & $\mathrm{O}-\mathrm{C} 2$ & $\mathrm{r}$ & se & $x$ pix & ypix \\
\hline 020101 & 1 & 2002 & 1 & 1.5899248 & 286 & 1 & 10 & 21.007 & 10.152 & 0.07 & 0.07 & 1 & 1 & -0.046 & -0.003 & 0 & 1 & -51.18 & -35.70 \\
\hline 020101 & 1 & 2002 & 1 & 1.5899248 & 286 & 1 & 20 & 38.084 & -3.194 & 0.04 & 0.04 & 1 & 1 & -0.048 & 0.002 & 0 & 1 & -101.93 & -7.68 \\
\hline 020101 & 1 & 2002 & 1 & 1.5899248 & 286 & 1 & 30 & -18.026 & -20.629 & 0.03 & 0.03 & 1 & 1 & -0.008 & -0.023 & 0 & 1 & 38.88 & 62.11 \\
\hline 020101 & 1 & 2002 & 1 & 1.5899248 & 286 & 1 & 40 & -62.693 & -6.437 & 0.04 & 0.04 & 1 & 1 & 0.015 & -0.024 & 0 & 1 & 162.85 & 43.52 \\
\hline 020101 & 1 & 2002 & 1 & 1.5899248 & 286 & 1 & 50 & 69.658 & -20.354 & 0.04 & 0.04 & 1 & 1 & -0.012 & -0.034 & 0 & 1 & -192.58 & 24.27 \\
\hline 020101 & 1 & 2002 & 1 & 1.5899248 & 286 & 1 & 60 & 64.711 & -81.411 & 0.04 & 0.04 & 1 & 1 & 0.008 & -0.052 & 0 & 1 & -205.37 & 187.62 \\
\hline 020101 & 1 & 2002 & 1 & 1.5899248 & 286 & 1 & $\mathrm{C} 0$ & -70.807 & 105.662 & 0.00 & 0.00 & 1 & 1 & 0.000 & 0.000 & 0 & 1 & 231.22 & -249.68 \\
\hline 020101 & 1 & 2002 & 1 & 1.5899248 & 286 & 0 & $\mathrm{C}^{*}$ & 67.869 & 20.090 & 0.00 & 0.00 & 1 & 0 & 99.999 & 99.999 & 0 & 1 & 9999.99 & 9999.99 \\
\hline 060207 & 359 & 2006 & 2 & 7.7272222 & 286 & 1 & 10 & -25.492 & -8.763 & 0.05 & 0.05 & 1 & 1 & 0.043 & 0.151 & 0 & 14 & 68.20 & 23.48 \\
\hline 060207 & 359 & 2006 & 2 & 7.7272222 & 286 & 1 & 20 & 25.9 & 12.8 & 0.03 & 0.03 & 1 & 1 & 0.013 & -0.012 & 0 & 14 & -69.31 & -34.52 \\
\hline 060207 & 359 & 2006 & 2 & 7.7272222 & 286 & 1 & 30 & 38.406 & 13.859 & 0.04 & 0.04 & 1 & 1 & -0.001 & -0.009 & 0 & 14 & -102.75 & -37.13 \\
\hline 060207 & 359 & 2006 & 2 & 7.7272222 & 286 & 1 & 40 & -7.702 & -21.809 & 0.03 & 0.03 & 1 & 1 & 0.008 & 0.005 & 0 & 14 & 20.58 & 58.36 \\
\hline 060207 & 359 & 2006 & 2 & 7.7272222 & 286 & 1 & 50 & -53.045 & 17.467 & 0.04 & 0.04 & 1 & 1 & 0.005 & -0.036 & 0 & 14 & 141.95 & -46.68 \\
\hline 060207 & 359 & 2006 & 2 & 7.7272222 & 286 & 1 & 60 & -177.778 & -52.242 & 0.05 & 0.05 & 1 & 1 & 0.019 & 0.087 & 0 & 14 & 475.67 & 139.93 \\
\hline 060207 & 359 & 2006 & 2 & 7.7272222 & 286 & 1 & $\mathrm{C} 0$ & -73.312 & 89.252 & 0.00 & 0.00 & 1 & 1 & 0.000 & 0.000 & 0 & 14 & 195.79 & -239.30 \\
\hline 060207 & 359 & 2006 & 2 & 7.7272222 & 286 & 0 & $\mathrm{C}^{*}$ & 129.555 & 19.230 & 0.00 & 0.00 & 1 & 0 & 99.999 & 99.999 & 0 & 14 & 9999.99 & 9999.99 \\
\hline
\end{tabular}

(This table is available in its entirety in machine-readable and Virtual Observatory (VO) forms in the online journal. A portion is shown here for guidance regarding its form and content.)

center point of a CCD frame. Besides, the center's $\mathrm{O}-\mathrm{C} 1$ and $\mathrm{O}-\mathrm{C} 2$ are designed 99.999 in the two directions, and 9999.99 for its two pixel coordinates, respectively. Finally, $\mathrm{O}-\mathrm{C} 1$ and $\mathrm{O}-\mathrm{C} 2$ in the line including $\mathrm{C} 0$ are designed 0.000 in the two directions.

\section{CONCLUSIONS}

2154 precise positions of Saturnian major satellites, measured relatively to Saturn from 359 CCD exposures taken by the $1 \mathrm{~m}$ telescopes at the Yunnan Observatory during the years 2002-2006, are presented in this paper. After our former image-processing techniques were improved for measuring pixel positions of both major satellites (especially for Mimas and Enceladus) and Saturn, and the four bright satellites-Tethys, Dione, Rhea and Titan — of Saturn were used to calibrate CCD FOV by comparing their pixel positions with their theoretical positions from the theory TASS1.7; the positions of all major satellites when measured with respect to Rhea usually had good agreement with their theoretical positions (TASS1.7) except for Mimas, which had the biggest systematic difference of about -0.3 arcsec in R.A. in its 2002 observational subsets. However, these differences became much smaller when the recent JPL ephemeris of Mimas was replaced. Our rms errors in each coordinate were about 40 mas for Saturn and its bright satellites S2-S6, and 90 mas for Mimas. These positional observations should be among the best ground-based CCD observations.
The authors thank the anonymous referee for his/her valuable comments to improve the scientific quality of this paper. They are also very grateful to the staff working at the $1 \mathrm{~m}$ telescope at the Yunnan Observatory for their assistance and support. This work was financially supported by the National Natural Science Foundation of China (Grants 10573008 and 10778617).

\section{REFERENCES}

Arlot, J.-E., \& Thuillot, W. 1993, Icarus, 105, 427

Colas, F. 1996, in Proc. of the PHESAT95 Workshop, Suppl. Ann. Phys., 21, 133

Duriez, L., \& Vienne, A. 1997, A\&A, 324, 366

Gonzalez, R. C., \& Woods, R. E. 1992, Digital Image Processing (Reading, MA: Addison-Wesley)

Harper, D., et al. 1997, A\&AS, 121, 65

Lindegren, L. 1980, A\&A, 89, 41

Pascu, D., et al. 1987, AJ, 93, 963

Peng, Q. Y. 2005, MNRAS, 359, 1597 (P1)

Peng, Q. Y., Vienne, A., \& Shen, K. X. 2002, A\&A, 383, 296

Peng, Q. Y., et al. 2003, A\&A, 401, 773

Qiao, R. C., Shen, K. X., \& Liu, J. R. 1999, A\&AS, 137, 1

Shen, K. X., Dourneau, G., Qiao, R. C., \& Liu, J. R. 2001, A\&A, 367,1061

Standish, E. M., Jr. 1998, JPL planetary and Lunar Ephemerides, DE405/LE405, JPL Interoffice Memo. 312.F-98-048.0. (Pasadena, CA: JPL)

Veiga, C. H., et al. 2003, A\&A, 400, 1095

Vienne, A., \& Duriez, L. 1995, A\&A, 297, 588

Vienne, A., et al. 2001a, A\&A, 380, 727

Vienne, A., Thuillot, A., \& Arlot, J.-E. 2001b, Note Sci. et Tech. de l'I.M.C.C.E., S077, 1 